\title{
A Scalable and Process Variation Aware NVM-FPGA Placement Algorithm
}

\author{
Chengmo Yang \\ University of Delaware \\ chengmo@udel.edu
}

\author{
Yuan Xue \\ University of Delaware \\ xueyuan@udel.edu
}

\begin{abstract}
As non-volatile memory (NVM) based FPGAs gain increasing popularity, FPGA synthesis tools start to tune the synthesis flow to match NVM characteristics. State-of-the-art NVM FPGA placement algorithms tried to reduce the high reconfiguration cost induced by the costly NVM programming process. However, they are not only limited in scalability but also fail to consider process variation. This paper aims to overcome these limitations. Blocks in the NVM-FPGA are no longer uniform but classified into fast, slow, and dead blocks. Moreover, the proposed placement algorithm reduces computation complexity by not searching the entire design space for an optimal solution with minimum reconfiguration cost, but computing the reconfiguration cost just-in-time. Verilog-to-Routing (VTR)-based implementation confirms its effectiveness in reducing critical path length and speeding up the placement process, while still saving reconfiguration cost by up to $74.2 \%$.
\end{abstract}

\section{CCS CONCEPTS}

- Hardware $\rightarrow$ Non-volatile memory; Placement; Emerging architectures.

\section{KEYWORDS}

FPGA, Non-volatile memories, Low power design, Placement

\section{ACM Reference Format:}

Chengmo Yang and Yuan Xue. 2019. A Scalable and Process Variation Aware NVM-FPGA Placement Algorithm. In Great Lakes Symposium on VLSI 2019 (GLSVLSI '19), May 9-11, 2019, Tysons Corner, VA, USA. ACM, New York, NY, USA, 6 pages. https://doi.org/10.1145/3299874.3317971

\section{INTRODUCTION}

FPGAs play an important role in IC design by offering high degrees of reconfigurability and self-adaptivity, a key request of many embedded and cyber-physical systems. However, traditional FPGAs cannot meet the rapidly growing needs of popular applications such as bioinformatics and neural networks, because their on-chip reconfigurable components are built with SRAMs which suffer from low cell density [1], high power dissipation [2], and low error resistance [3]. To overcome these limitations, researchers have proposed the use of Non-Volatile Memories (NVM) in FPGAs, and

Permission to make digital or hard copies of all or part of this work for personal or classroom use is granted without fee provided that copies are not made or distributed for profit or commercial advantage and that copies bear this notice and the full citation on the first page. Copyrights for components of this work owned by others than ACM must be honored. Abstracting with credit is permitted. To copy otherwise, or republish, to post on servers or to redistribute to lists, requires prior specific permission and/or a fee. Request permissions from permissions@acm.org.

GLSVLSI '19, May 9-11, 2019, Tysons Corner, VA, USA

(c) 2019 Association for Computing Machinery.

ACM ISBN 978-1-4503-6252-8/19/05 . .\$15.00

https://doi.org/10.1145/3299874.3317971 demonstrated the feasibility of building on-chip reconfigurable components with Spin-Transfer Torque Magnetic RAM (STT-RAM) [4], Resistive RAM (RRAM)[5], and Phase Change Memory (PCM) [6], to name a few. These NVM-FPGAs are expected to offer more reconfigurable resources, consume less power, and be more resilient to power supply interruptions.

Despite the aforementioned advantages, NVMs are also known for their shortcomings of slow write speed, high write energy, and limited endurance [7]. To mitigate the potential adverse impact on FPGA reconfiguration latency, energy, and programming cycles, a number of synthesis optimizations have been proposed for NVMFPGAs. Specially, NVM-friendly placement techniques $[8,9]$ have been proposed to reduce the reconfiguration cost, represented as the number of NVM cells programmed during reconfiguration. While effective, these techniques search the entire design space for an optimal solution [8], thus tremendously increasing the execution time of the placement algorithm. Another limitation is that previous NVM-FPGA synthesis optimizations ignore process variation (PV) during fabrication. When technology scales below 65nm [10], PV causes high variations in the attributes of devices and wires, which in turn lead to significant variations in delay, power, and aging speed of different FPGA on-chip components. The PV influence on NVM-FPGAs is even more severe than SRAM-FPGAs, as the feature size of NVM is smaller and the technology is less mature [11].

This work aims to tackle the limitations of previous NVM-FPGA synthesis optimizations, by proposing a more scalable and process variation aware NVM-FPGA placement framework. First, onchip reconfiguration components in the NVM-FPGA are no longer treated uniformly but classified into fast, slow, and dead blocks based on PV information. Such classification is performed repetitively throughout the whole FPGA lifetime to account for the impact of aging. A block variation-aware placement region selection approach is proposed for selecting a minimum-size region with sufficient number of fast blocks for a given design. Second, a scalable NVM-FPGA placement algorithm is proposed, which computes reconfiguration cost just-in-time and drastically speeds up the placement process, making it more suitable for large designs than the state-of-the-art NVM-FPGA placement algorithm [8]. Overall, this work makes the following contributions.

- Development of non-uniform block delay models and a corresponding placement region selection algorithm to account for PV and aging.

- Design of a scalable placement algorithm that balances reconfiguration cost and timing while reducing placement overhead.

- Incorporation of the proposed NVM-FPGA framework into the Verilog-to-Routing (VTR) CAD tool [12] for evaluation. 
In the rest of this paper, Section 2 reviews background knowledge of NVM-FPGAs and their synthesis optimizations, as well as process variation and aging issues. Section 3 describes the proposed placement region selection and scalable placement algorithm. Section 4 presents the experimental setup and results, while Section 5 concludes the paper.

\section{BACKGROUND}

NVM-FPGAs: FPGAs typically include three types of on-chip reconfigurable resources: configurable logic blocks (CLBs), connection blocks (CBs), and switch boxes (SBs). CLBs are used to implement logic functions. In each CLB there is a cluster of basic logic elements (BLEs), each of which is made of one look up table (LUT) and two flip-flops. SBs are used for routing, and CBs are used to connect CLBs and SBs locally.

Configuration of each CLB, SB, and CB is controlled by on-chip memory cells which can be implemented with NVM cells. Various NVM-FPGA architectures have been developed/proposed. Actel has taped out Flash-based FPGAs [13]. [6] proposed 3D architectures for PCM-based FPGAs. The NVM-FPGA proposed in [4] utilizes STT-RAM, a magnetic storage element, as the basic memory cell. RRAM based FPGAs are proposed in [5]. These efforts prove the feasibility of NVM-FPGAs.

NVM-FPGA Synthesis: Recently, researchers started to work on tuning the FPGA synthesis flow to fit NVM characteristics. In [14], a multi-context switching framework is proposed for Racetrack memory based FPGA, to speed up reconfiguration and save power. Placement algorithms are proposed in [8] to reduce the reconfiguration cost of CLBs for NVM-FPGAs. In [15], an algorithm to reuse paths during routing and minimize reconfiguration writes to SBs is proposed. In [9], a runtime and reconfiguration dual aware placement strategy is proposed for a SRAM-NVM hybrid FPGA architecture. In [16], a memory and logic co-placement is proposed for balancing the write distribution across memory and logic blocks in RRAM-FPGAs. However, none of these previous synthesis optimizations have considered process variation, which is one of the main focus of this work.

Technically, the proposed work is most closely related with [8] in that both of them aim to reduce CLB reconfiguration cost through placement optimizations. However, the algorithms in [8] explore the entire design space offline to find an optimization solution with minimum reconfiguration cost. As a result, they are unscalable and only suitable for relatively small designs. In contrast, this work computes CLB reconfiguration cost just-in-time during the placement stage, and hence is more scalable and supports larger designs.

Process Variation Impact: Process variation (PV) is the natural occurrence in deep sub-micron and nano-scale technologies. With PV, critical parameters such as channel length, width, and oxide thickness shift from designed values. Common PV includes random dopant fluctuation (RDF), line-edge and linewidth roughness (LER, LWR), oxide thickness variations, defects and traps, patterning proximity effect, to name a few [17].

NVM devices are more vulnerable to PV than SRAM as the feature size of NVM is expected to be smaller and the technology is less mature [11]. For example, thicker GST $\left(\mathrm{Ge}_{2} \mathrm{Sb}_{2} \mathrm{Te} e_{5}\right)$ layer in PCM will require higher programming current [18], which may in turn lead to higher power consumption and reduced programming cycles, while the over-aging element would have larger access delay and lower reliability. It is shown in [18] that PV could increase PCM programming power by $96 \%$ and shorten PCM endurance by $50 \mathrm{X}$. Some recent works have considered process variation of NVM devices. For example, the work in [19] considers PV-induced reliability degradation for Flash-based storage systems and proposes a read improvement scheme. A wear-leveling technique for multi-level PCM is proposed in [20] with process variation considerations.

PV leads to significant variations in read and write speed, power, and aging speed of different reconfiguration components of an FPGA. In [21], a statistical simulation method is introduced to study PV's adverse effect on the stability of configuration memory. In [10], a degradation model considering PV is constructed and the corresponding evaluation method is given. In [22], a two-phase placement algorithm is proposed to maximize design reliability under PV when voltage scaling is applied. All these works target SRAM-based FPGAs and focus on the configuration memory. In contrast, the proposed work targets NVM-based FPGAs and considers PV's impact on CLBs during placement.

\section{PROPOSED PLACEMENT FRAMEWORK}

In a nutshell, this work targets the placement stage on the synthesis flow and hence focuses on CLBs which are the only type of blocks configured during placement. It introduces two steps to the conventional synthesis flow, namely, process variation $(P V)$ aware region selection and scalable NVM-FPGA placement, to be applied after packing and before routing. The goals are reducing CLB reconfiguration cost as well as improving performance by reducing critical path delay.

\subsection{PV-Aware Placement Region Selection}

3.1.1 Block classification. Block variation comes from two factors: the initial PV distribution introduced during fabrication, as well as aging, reflected as the programming cycles of various CLBs.

Block delay and reliability can be measured with some existing approaches, such as the delay variation monitor approach in [23] and the fault detection method in [24]. CLBs with stuck-at faults (either worn out or due to fabrication flaws) are marked as "dead blocks" and will no longer be used. For the remaining fault-free CLBs, their access delays (i.e., read speed) will be measured and a user defined ratio $\alpha$ will be employed to differentiate fast and slow blocks on the FPGA. This proposed model is generic and can be extended to include other FPGA blocks (i.e., SBs and CBs) as well. Figure 1 presents an example of block classification.

$$
\alpha=\frac{\text { fast block count }}{\text { total block count }}
$$

To account for the impact of aging, the delay and reliability measurement takes place repetitively throughout the FPGA lifetime, e.g., upon placing $n$ designs. A new measurement can be performed during the gap period between configuring two designs to completely hide its overhead. In a new measurement, a fast block may become a slow block while a slow block may end up as a dead block.

3.1.2 Placement region selection. Traditionally, a placement region is a square (or near-square) region [12] located at one fixed corner of 


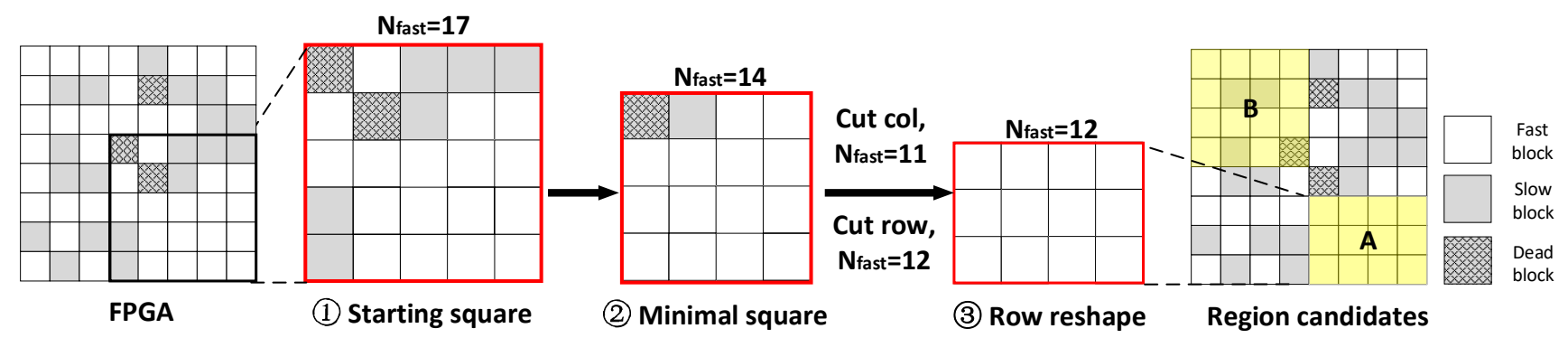

Figure 1: A region selection example. Design block number $N=11$, fast block ratio $\alpha=0.65$. The bottom right corner region is selected in three steps. Region candidate $A$ is better than $B$.

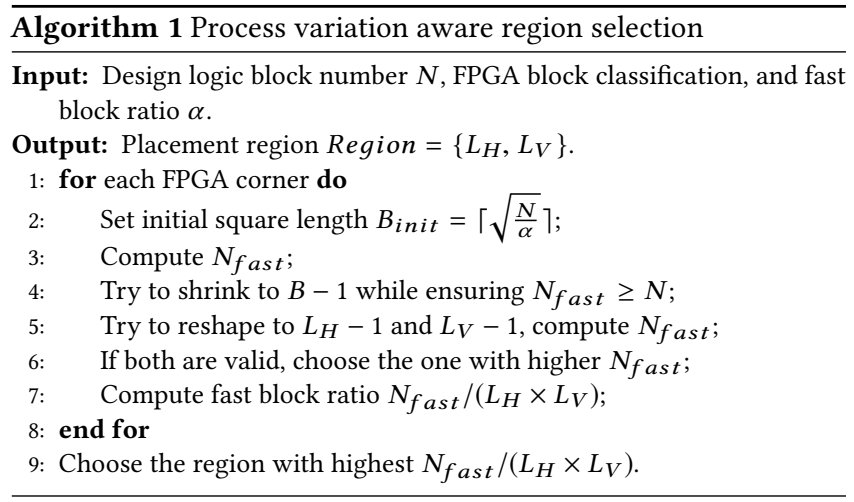

the FPGA, as this shape and position tend to minimize critical path delay, improve routability, and reduces the distances to I/O pins. Instead of using a fixed location and shape, the proposed region selection scheme searches all four corners of the FPGA, finds four candidates, and chooses the best region among the four candidates, as sketched in Algorithm 1. It imposes an extra criterion, that is, the number of fast blocks in the selected region (denoted as $N_{\text {fast }}$ ) must be sufficient to hold the design which requires $N$ CLBs:

$$
N_{\text {fast }} \geq N
$$

To search for a minimum region that satisfies inequality (2), the algorithm first searches for the minimal square (lines 2-4). Assume a placement region has horizontal length $L_{H}$ and vertical length $L_{V}$. If $N_{\text {fast }}$ in the region satisfies inequality (2), the algorithm tries to shrink both $L_{H}$ and $L_{V}$ by one. The new $N_{f a s t}$ can be easily computed by subtracting the fast block count on the removed row and column. If the reduced region does not meet inequality (2), the minimum square length $B_{\text {min }}$ is obtained. In the next step, the algorithm tries to reshape the region to the minimal near-square, by removing one row or one column on the boundary and checking if the reshaped region still satisfies inequality (2). If both row removing and column removing satisfy the constraint, the solution with more remaining fast blocks will be used.

Figure 1 illustrates the region search process with an example. Assume the design requires $N=11 \mathrm{CLBs}$, and the average ratio of fast blocks $\alpha=0.65$. The initial square length $L_{V}=L_{H}=\lceil\sqrt{11 / 0.65}\rceil=5$. Within this region $N_{\text {fast }}=17>N$. Then the square is shrinked to $L_{V}=L_{H}=4$, and $N_{\text {fast }}=14>N$. However, if the square is shrinked to $L_{V}=L_{H}=3$, we will have $N_{\text {fast }}<N$, thus $4 \times 4$ is the minimal square. Step 3 shows that both row reshape and colomn reshape are possible, but row reshape leads to more remaining fast blocks $N_{\text {fast }}=12$. Therefore the $3 \times 4$ region becomes the selected region candidate, shown as region A on the FPGA.

Although the region selection process works at any location on the FPGA, it is performed at each corner so as to minimize the distances to I/O pins. Four placement region candidates will be obtained, and the one with the smallest size and highest ratio of fast blocks $N_{\text {fast }} /\left(L_{H} \times L_{V}\right)$ will be chosen (line 9 of Algorithm 1). For example, in Figure 1, region A is selected as it is smaller than region $\mathrm{B}$ and has $100 \%$ fast blocks.

\subsection{Scalable NVM-FPGA Placement}

After selecting an appropriate region with a sufficient number of fast blocks for the given design, the scalable NVM-FPGA placement takes place within the selected region. This section first introduces the proposed NVM-FPGA placement algorithm and then compares it with the state-of-the-art NVM-FPGA placement [8] in terms of complexity to highlight its advantages.

3.2.1 Scalable Placement Algorithm. A generic FPGA placement flow is composed of four major steps. First, an initial placement is generated as the starting point. Then, the algorithm enters the iterative stage. At each iteration, two blocks are randomly selected to form a swapping trial, which is either accepted or rejected based on pre-defined acceptance conditions. Finally, the algorithm checks whether the current placement process meets the termination condition. The proposed placement algorithm differs from the state-ofthe-art NVM-FPGA placement [8] in initial placement generation, swapping block selection, and swapping acceptance condition.

First, the initial placement in the state-of-the-art placement is a globally optimal min-bit-flip (MBF) placement with minimum reconfiguration cost [8]. Such cost is computed bit-by-bit as most NVM-FPGA optimizations employ a read-before-write strategy to reduce NVM writes at the bit level. However, it is unnecessary to start with a time-consuming optimal mapping because the later swapping trials which aim to balance timing, congestion, and reconfiguration cost will destroy the optimality in reconfiguration cost anyway. As a result, the proposed algorithm starts not with the MBF placement but with a random placement, generated by randomly placing all the CLBs on the fast blocks in the selected placement region. This change largely reduces the complexity of the placement algorithm without noticeably degrading its quality. 


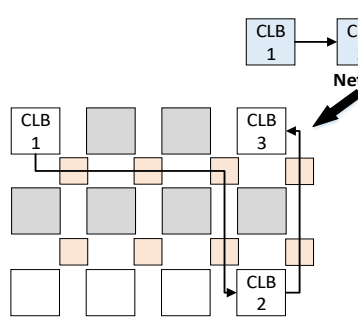

(a) Fast Only

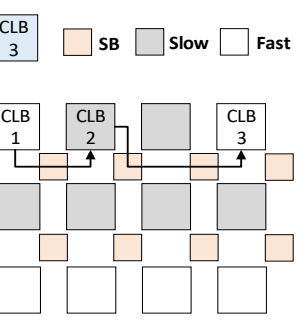

(b) Fast+Slow
Figure 2: Benefit of using slow blocks. Block delay becomes longer, but the path length is largely shortened.

Second, the proposed placement differs from the state-of-the-art in that it considers process variation across different blocks. While the initial placement only uses fast blocks, at the block swapping stage the algorithm allows the use of slow blocks as it offers more placement flexibilities in terms of timing and routablility considerations. Figure 2 shows a sample design with three CLBs and two paths, CLB1 $\rightarrow$ CLB2 and CLB2 $\rightarrow$ CLB3. The placement region has six slow blocks and six fast blocks. Figure 2(a) shows a placement that uses only fast blocks, while in Figure 2(b) the position of CLB2 is swapped to a slow block. This swapping is beneficial because although the block delay of CLB2 becomes longer, the two paths are largely shortened. For this reason, the proposed algorithm allows fast to slow block swapping.

Regarding the swapping acceptance condition, the state-of-theart uses a cost function to integrates timing, congestion, and reconfiguration cost, and accepts a swapping trial only if the cost becomes lower (i.e., $\Delta$ Cost $<0$ ), indicating that the swapping is beneficial. This greedy strategy may end up sticking at a local optimal point. To avoid this undesired case and explore globally for more possible solutions, the proposed algorithm adopts simulated annealing [25], which accepts a swapping trial not only when $\Delta$ Cost $<0$ but also if $e<r^{-\frac{\Delta \operatorname{Cos} t}{T}}$, wherein $r$ is a random value in $(0,1)$ and $T$ is the temperature. $T$ decreases as the algorithm proceeds, allowing it to eventually converge to a near-optimal point.

3.2.2 Placement complexity. The design space of reconfiguration cost includes three types of flexibilities [8], as illustrated in Figure 3. Assume a design requires $m$ CLBs, the selected placement region includes $n$ CLBs, each CLB contains $v$ LUTs, and each LUT has $k$ inputs among which $l$ are used. The whole design space is:

$$
\text { Flex }_{\text {Design }}=\left(2^{k-l} \times \frac{k !}{(k-l) !}\right) \times(v !) \times\left(\frac{n !}{(n-m) !}\right)
$$

State-of-the-art NVM-FPGA placement algorithm [8] explores all the three types of flexibilities to generate a globally optimal $\mathrm{MBF}$ placement and use it as an initial placement. Its steps and complexity are illustrated in Figure 3. Specifically, it first computes a CLB level reconfiguration cost matrix $R_{C L B}[m][n]$, and then applies the KM algorithm [26] with $O\left(m n^{2}\right)$ complexity to solve the optimal mapping problem. However, computing $R_{C L B}$ is time consuming as each of the $m n$ elements in the matrix is the cost of an optimal mapping at the CLB level, shown as $C_{l u t}$ in Figure 3. Generation of $C_{l u t}$ consists of KM mapping cost $O\left(v^{3}\right)$ and matrix $R_{L U T}[v][v]$ construction cost. Similarly, each of the $v^{2}$ elements in $R_{L U T}$ is the cost of an optimal mapping at the input level, shown as $C_{\text {input }}$

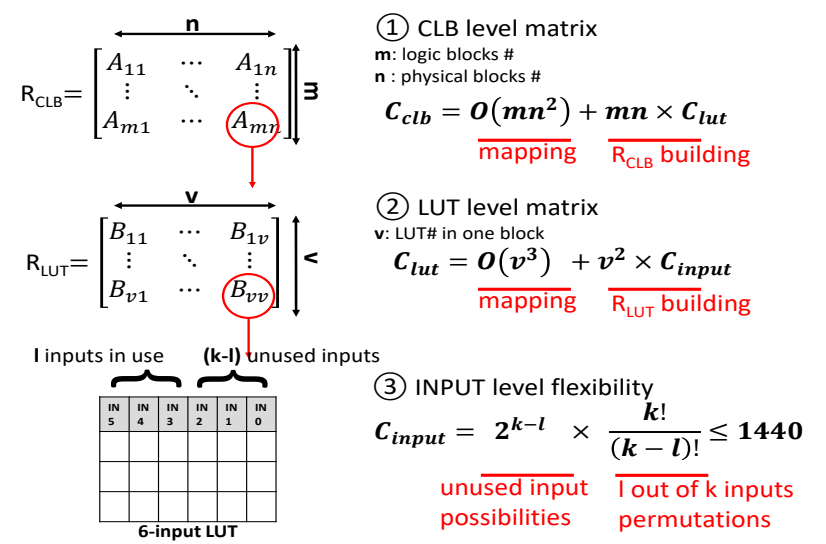

Figure 3: State-of-the-art placement complexity

in Figure 3. To compute $C_{\text {input }}$, the input level flexibility is fully explored by numerating all possibilities. Each of the $k-l$ unused inputs have two possible values ( 0 and 1 ), while the $l$ used inputs have full permutations. For the standard setting of 6-input LUT $(k=6)$, the highest possible value of $C_{\text {input }}$ is 1440 , reached when $l$ is 4 or 5 . Considering these three levels of complexities, the overall complexity $C_{\text {design }}$ of the state-of-the-art MBF placement is:

$$
\begin{aligned}
C_{\text {design }} & =O\left(m n^{2}\right)+m n \times\left[O\left(v^{3}\right)+v^{2} \times 1440\right] \\
& =O\left(m n^{2}\right)+O\left(m n v^{3}\right)+1440 \times O\left(m n v^{2}\right)
\end{aligned}
$$

The proposed placement algorithm reduces the complexity from two perspectives. First, it starts not with a globally optimal MBF placement but with a random placement, thus completely eliminating the CLB-level overhead (term $O\left(m n^{2}\right)$ in Equation (4)). This also implies that the CLB-level reconfiguration cost matrix $R_{C L B}[m][n]$ does not need to be fully constructed $a$ priori but can be computed just-in-time during swapping trials. Specifically, an element $R_{C L B}[i][j]$ is computed only if a swapping trial decides to map $C L B_{i}$ to $B L K_{j}$ and if $R_{C L B}[i][j]$ has not been computed yet. Assume $\delta$ is the sparsity of the matrix, the total number of calculated elements in $R_{C L B}[m][n]$ is $\delta \times m n$.

Second, as shown in Equation (4)), the most time consuming part of the placement procedure is the exploration of input-level flexibility $\left(1440 \times O\left(m n v^{2}\right)\right)$. In the proposed algorithm, such flexibility is not explored in block swapping trials. Instead, direct input mapping is taken when computing $R_{L U T}$, and hence $C_{i n p u t}=1$. Later upon finishing CLB-level and LUT-level mappings, input-level flexibility will be explored. The complexity is $1440 \times m v$ since $m \times v$ LUTs are used in total and each LUT requires up to 1440 trials to find the optimal input-level mapping. The overall complexity of the proposed placement is:

$$
\begin{aligned}
C_{\text {design }}^{\prime} & =\delta \times m n \times\left[O\left(v^{3}\right)+v^{2} \times 1\right]+m v \times 1440 \\
& =O\left(\delta m n v^{3}\right)+O\left(\delta m n v^{2}\right)+1440 \times m v
\end{aligned}
$$

A comparison between Equations (4) and (5) shows that the proposed placement algorithm improves the state-of-the-art by completely eliminating CLB level-complexity, reducing LUT-level complexity by $1 / \delta$ times, and reducing input-level complexity by $n v$ times. 
Table 1: Benchmark circuits

\begin{tabular}{|c|l|r|r|}
\hline No & Benchmark & CLB\# & LUT\# \\
\hline 1 & stereovision1 & 1365 & 13066 \\
2 & stereovision0 & 1479 & 14622 \\
3 & LU8PEEng & 2583 & 21954 \\
4 & bgm & 3419 & 30089 \\
5 & stereovision2 & 3635 & 36341 \\
6 & LU32PEEng & 8861 & 88608 \\
7 & mcml & 10436 & 104066 \\
\hline
\end{tabular}

Table 2: Experimental configurations in use

\begin{tabular}{|c|l||c|c|c|c|}
\hline No & Schemes & PV & Region & Placement & Block types \\
\hline 1 & VTR & - & - & VTR & Fast \\
2 & VTR+PV & $\checkmark$ & - & VTR & Fast+Slow \\
3 & VTR+PVRS+F & $\checkmark$ & $\checkmark$ & VTR & Fast \\
4 & VTR+PVRS & $\checkmark$ & $\checkmark$ & VTR & Fast+Slow \\
5 & R\&T+PVRS & $\checkmark$ & $\checkmark$ & R\&T & Fast+Slow \\
6 & Proposed & $\checkmark$ & $\checkmark$ & Proposed & Fast+Slow \\
\hline
\end{tabular}

\section{EXPERIMENTAL EVALUATION}

\subsection{Experimental Methodology}

The proposed placement framework is incorporated into the VTR 7.0 CAD flow [12] for evaluating its impact on placement complexity, design timing, and NVM-FPGA endurance. The experimental FPGA architecture is Stratix IV GX, with a $40 \mathrm{~nm}$ fabrication library. Each CLB consists of 10 LUTs, and each LUT has 6 inputs. A simulator written in $\mathrm{C}$ is used to simulate PV on the FPGA. Normal distribution is applied. The overall fast block ratio $\alpha=0.8$. The delay models in VTR 7.0 have been modified to reflect the differences in the three types of blocks. Fast blocks still use the standard delay model, while slow blocks are set to be $50 \%$ slower than the standard model based on the results of [9]. The delay of dead block is set to infinite.

The largest benchmarks offered by VTR (with 1000+ CLBs) are selected to evaluate the complexity of the proposed algorithm. Benchmark information is listed in Table 1, ranked by design size in ascending order. Six experimental configurations are evaluated, as listed in Table 2. Each configuration is defined by four metrics: process variation $(\mathrm{PV})$, placement region selection, the placement algorithm, and the type of blocks used.

\subsection{PV Aware Region Selection Evaluation}

Our first set of experiments evaluates the proposed PV aware region selection in terms of timing. The first four configurations in Table 2 are compared. "VTR" is the original VTR placement without considering PV in the FPGA. "VTR+PV" considers PV but does not perform region selection. "VTR+PVRS+F" and "VTR+PVRS" perform $\mathrm{PV}$-aware placement region selection. The former uses only fast blocks, while the latter uses both fast and slow blocks.

The results of critical path delay are shown in Table 3. "VTR" serves as the baseline and raw data are displayed, while ratios over the baseline are given for the other three configurations. "VTR+PV" demonstrates the necessity for considering $\mathrm{PV}$ in placement. If $\mathrm{PV}$ is ignored, the critical path delay is severely increased by $22.7 \%$ on average. "VTR+PVRS" and "VTR+PVRS+F" demonstrate the effectiveness of the proposed region selection algorithm in overcoming
Table 3: Critical path delay of three configurations

\begin{tabular}{|l|r|r|r|r|}
\hline Benchmark & $\begin{array}{r}\text { VTR } \\
\text { (ns) }\end{array}$ & $\begin{array}{r}\text { VTR+PV } \\
\text { (ratio) }\end{array}$ & $\begin{array}{r}\text { VTR+PVRS+F } \\
\text { (ratio) }\end{array}$ & $\begin{array}{r}\text { VTR+PVRS } \\
\text { (ratio) }\end{array}$ \\
\hline stereovision1 & 5.608 & $130.8 \%$ & $100.0 \%$ & $100.0 \%$ \\
stereovision0 & 4.438 & $132.0 \%$ & $100.0 \%$ & $100.0 \%$ \\
LU8 & 146.186 & $109.5 \%$ & $101.5 \%$ & $99.6 \%$ \\
bgm & 27.392 & $128.3 \%$ & $110.4 \%$ & $106.1 \%$ \\
stereovision2 & 15.281 & $129.5 \%$ & $118.4 \%$ & $110.8 \%$ \\
LU32 & 147.109 & $118.5 \%$ & $105.2 \%$ & $105.1 \%$ \\
mcml & 106.523 & $109.9 \%$ & $107.8 \%$ & $107.2 \%$ \\
\hline AVE & - & $122.7 \%$ & $106.2 \%$ & $104.1 \%$ \\
\hline
\end{tabular}

PV introduced block performance degradation in real chips. They only slightly increase the critical path delay by $4.1 \%$ and $6.2 \%$ on overage. "VTR+PVRS" outperforms "VTR+PVRS+F" with $2.1 \%$ timing improvement, which comes from the opportunities provided by slow blocks in shortening path delays, and is consistent in each benchmark. Accordingly, "VTR+PVRS" will be used as the baseline for the study of placement algorithms.

\subsection{Scalable Placement Evaluation}

Our second set of experiments evaluates the proposed placement algorithm in term of its complexity and its impact on NVM-FPGA endurance and design timing. It is compared with the baseline VTR placement and the state-of-the-art NVM-FPGA placement "R\&T" [8]. For a fair comparison, all the placement algorithms are applied in the selected placement region and they all use both fast and slow blocks. To create the reconfiguration scenario similar with [8], six test pairs (TP) are constructed from the benchmarks in Table 1. TP $i$ tries to configure benchmark $i$ on the FPGA with benchmark $i+1$ being the existing content. For example, TP1 is to configure "stereovision1" on the FPGA by modifying existing design "stereovision0".

Table 4 shows a comprehensive comparison among different placement techniques. "VTR+PVRS" is used as the baseline for which raw data are presented, while ratios over the baseline are given for "R\&T+PVRS" and "Proposed".

4.3.1 Complexity evaluation. The sparsity $\delta$ of the reconfiguration matrix $R_{C L B}[m][n]$ is evaluated to show the efficiency of just-intime reconfiguration cost computation. As shown in Table 4, most of the test cases have less than 0.5 sparsity. TP6 has the lowest sparsity of 0.302 . This confirms the complexity advantage of computing $R_{C L B}$ elements only when necessary.

The execution time of different algorithms is compared, also shown in Table 4. The execution time of "VTR+PVRS" is mainly spent on performing placement trials. It clearly shows an increasing trend as the design size increases. For "R\&T+PVRS", its execution time is composed of two parts, the time in generating the initial MBF placement, and the time in performing placement trials. With a larger design size, the execution time of "R\&T+PVRS" increases superlinearly; TP1 execution time is $1.38 \mathrm{X}$ of "VTR+PVRS", while TP6 execution time is $1.89 \mathrm{X}$ of "VTR+PVRS". This reflects the complexity of generating MBF placement, which increases by a factor of $O\left(n^{3}\right)$ theoretically. For large designs, the overhead for generating MBF would be overwhelming. In comparison, "Proposed" integrates reconfiguration cost computation into the placement trial process. 
Table 4: Execution time, reconfiguration cost, and critical path delay of three placements

\begin{tabular}{|c|c|c|c|c|c|c|c|c|c|c|}
\hline & \multirow[b]{2}{*}{$\delta$} & \multicolumn{3}{|c|}{ Execution time } & \multicolumn{3}{|c|}{ Reconfiguration cost } & \multicolumn{3}{|c|}{ Critical path delay } \\
\hline & & $\begin{array}{r}\text { VTR+PVRS } \\
\text { (second) }\end{array}$ & $\begin{array}{r}\text { R\&T+PVRS } \\
\text { (ratio) }\end{array}$ & $\begin{array}{r}\text { Proposed } \\
\text { (ratio) }\end{array}$ & $\begin{array}{r}\text { VTR+PVRS } \\
\text { (bit-flip count) }\end{array}$ & $\begin{array}{r}\text { R\&T+PVRS } \\
\text { (ratio) }\end{array}$ & $\begin{array}{r}\text { Proposed } \\
\text { (ratio) }\end{array}$ & $\begin{array}{r}\text { VTR+PVRS } \\
\text { (ns) }\end{array}$ & $\begin{array}{r}\text { R\&T+PVRS } \\
\text { (ratio) }\end{array}$ & $\begin{array}{r}\text { Proposed } \\
\text { (ratio) }\end{array}$ \\
\hline TP1 & 0.666 & 210.8 & $138.0 \%$ & $105.3 \%$ & $2.91 \mathrm{E}+05$ & $45.9 \%$ & $48.3 \%$ & 5.608 & $101.9 \%$ & $101.7 \%$ \\
\hline $\mathrm{TP} 2$ & 0.423 & 219.2 & $142.0 \%$ & $90.0 \%$ & $4.04 \mathrm{E}+05$ & $38.6 \%$ & $38.9 \%$ & 4.438 & $126.2 \%$ & $119.5 \%$ \\
\hline TP3 & 0.514 & 755.4 & $144.0 \%$ & $100.0 \%$ & $7.86 \mathrm{E}+05$ & $41.0 \%$ & $68.2 \%$ & 145.647 & $106.6 \%$ & $105.6 \%$ \\
\hline $\mathrm{TP} 4$ & 0.403 & 1107.1 & $160.3 \%$ & $101.7 \%$ & $9.62 \mathrm{E}+05$ & $72.3 \%$ & $82.8 \%$ & 29.053 & $103.8 \%$ & $106.5 \%$ \\
\hline TP5 & 0.418 & 965.1 & $167.0 \%$ & $98.8 \%$ & $8.77 \mathrm{E}+05$ & $25.7 \%$ & $25.8 \%$ & 16.934 & $107.5 \%$ & $107.6 \%$ \\
\hline TP6 & 0.302 & 7737.9 & $189.6 \%$ & $99.2 \%$ & $2.30 \mathrm{E}+06$ & $69.5 \%$ & $66.3 \%$ & 154.602 & $109.6 \%$ & $109.8 \%$ \\
\hline AVE & 0.454 & - & $156.8 \%$ & $99.1 \%$ & - & $48.8 \%$ & $55.1 \%$ & - & $109.3 \%$ & $108.4 \%$ \\
\hline
\end{tabular}

As the number of swapping trials is more-or-less stable, its execution time is close to "VTR+PVRS" even for large designs. The slight fluctuation is due to variations in swapping trial number and the difference in reconfiguration matrix sparsity.

Overall, this study confirms that the proposed placement is much more scalable than the state-of-the-art NVM-FPGA placement.

4.3.2 Performance evaluation. Table 4 also compares the performance of three placement schemes in terms of reconfiguration cost (i.e., the number of NVM writes) and timing. For reconfiguration cost, "R\&T+PVRS" achieves the lowest reconfiguration cost of $48.8 \%$ on average. This is because the algorithm is based on minimal reconfiguration cost placement "MBF" and it controls the tradeoff process so that the final reconfiguration cost is bounded to MBF. In comparison, the reconfiguration cost of "Proposed" is not bounded to MBF because both the initial placement and swapping trials are randomly generated. While "Proposed" outperforms "R\&T+PVRS" for some test cases such as TP6, its average reconfiguration cost is $6.3 \%$ more than "R\&T+PVRS".

For critical path delay, "R\&T+PVRS" shows a 9.3\% overhead and "Proposed" only has $8.4 \%$ overhead on average. "Proposed" achieves slightly better timing because the weight of timing cost is constant in its cost model (which is used for evaluating swapping trials). However, "R\&T" gradually reduces the weight of timing cost to prioritize reconfiguration cost towards the end of placement.

Overall, for the proposed NVM-FPGA algorithm, the small loss in reconfiguration cost is acceptable considering its better timing and significant advantage in complexity.

\section{CONCLUSIONS}

A scalable and process variation (PV) aware NVM-FPGA placement framework has been proposed in this paper. Blocks in the FPGA are classified into three types to model the impact of $\mathrm{PV}$, and the proposed framework chooses a qualified placement region considering block variations. Furthermore, a scalable NVM-FPGA placement has been developed to balance reconfiguration cost and timing, with dramatically reduced complexity compared with state-of-theart schemes. Experiments based on large VTR benchmarks show that the proposed scheme effectively balances reconfiguration cost and timing without introducing noticeable execution time overhead on top of VTR placement. This work potentially increases the popularity and practicality of NVM-FPGAs.

\section{ACKNOWLEDGMENT}

This work is partially supported by NSF Award \#1527464.

\section{REFERENCES}

[1] G. Dai, Y. Chi, Y. Wang, and H. Yang, "FPGP: Graph processing framework on FPGA a case study of breadth-first search," in FPGA, pp. 105-110, 2016.

[2] C.-H. Chen, P.-C. Hsiu, T.-W. Kuo, C.-L. Yang, and C.-Y. M. Wang, "Age-based PCM wear leveling with nearly zero search cost," in DAC, pp. 453-458, 2012.

[3] Actel, Understanding soft and firm errors in semiconductor devices, 2002.

[4] W. Zhao, E. Belhaire, C. Chappert, and P. Mazoyer, "Spin transfer torque STTMRAM-based runtime reconfiguration FPGA circuit," TECS, vol. 9, Oct. 2009.

[5] X. Tang, G. Kim, P.-E. Gaillardon, and G. D. Micheli, "A study on the programming structures for RRAM-based FPGA architectures," TCS I, vol. 63, Apr. 2016.

[6] Y. Chen, J. Zhao, and Y. Xie, "3D-nonfar: Three-dimensional non-volatile FPGA architecture using phase change memory," in ISLPED, pp. 55-60, 2010.

[7] http://www.itrs2.net/2013-itrs.html, ITRS, 2013.

[8] Y. Xue, P. Cronin, C. Yang, and J. Hu, "Fine-tuning CLB placement to speed up reconfigurations in NVM-based FPGAs," in FPL, pp. 1-8, 2015.

[9] Q. Lou, M. Zhao, L. Ju, C. J. Xue, J. Hu, and Z. Jia, "Runtime and reconfiguration dual-aware placement for SRAM-NVM hybrid FPGAs," in NVMSA, pp. 1-6, 2017.

[10] M. Yabuuchi and K. Kobayashi, "Evaluation of FPGA design guardband caused by inhomogeneous NBTI degradation considering process variations," in FPT, pp. 1-6, 2010.

[11] L. Shi, Y. Di, M. Zhao, C. J. Xue, K. Wu, and E. H.-M. Sha, "Exploiting process variation for write performance improvement on NAND flash memory storage systems," TVLSI, vol. 24, Feb. 2015.

[12] J. Luu, J. Goeders, M. Wainberg, A. Somerville, T. Yu, K. Nasartschuk, M. Nasr, S. Wang, T. Liu, N. Ahmed, K. B. Kent, J. Anderson, J. Rose, and V. Betz, "VTR 7.0: Next Generation Architecture and CAD System for FPGAs," TRTS, vol. 7, Jun. 2014.

[13] www.actel.com/documents/PA3HB.pdf, Proasic3 flash family FPGAs handbook, Actel, 2011.

[14] K. Huang, R. Zhao, and Y. Lian, "Racetrack memory-based nonvolatile storage elements for multicontext FPGAs," TVLSI, vol. 25, Sep. 2015.

[15] Y. Xue, P. Cronin, C. Yang, and J. Hu, "Routing path reuse maximization for efficient NV-FPGA reconfiguration," in ASP-DAC, pp. 360-365, 2016.

[16] Y. Xue, C. Yang, and J. Hu, "Age-aware logic and memory co-placement for RRAM-FPGAs," in DAC, pp. 1-6, 2017.

[17] K. Kuhn, "Managing process variation in Intel's 45nm CMOS technology," Intel Technology fournal, vol. 12, Jun. 2008.

[18] W. Zhang and T. Li, "Characterizing and mitigating the impact of process variations on phase change based memory systems," in MICRO, pp. 1-12, 2009.

[19] Q. Li, L. Shi, Y. Di, Y. Du, C. J. Xue, and E. H. Sha, "Exploiting process variation for read performance improvement on LDPC based flash memory storage systems," in ICCD, pp. 681-684, 2017.

[20] M. Zhao, L. Jiang, Y. Zhang, and C. J. Xue, "SLC-enabled wear leveling for MLC PCM considering process variation," in DAC, pp. 1-6, 2014.

[21] Y. Xu, L. Liu, M. Chan, and J. Watt, "Process variation impact on FPGA configuration memory," in ISQED, pp. 613-616, 2009.

[22] S. Golshan, A. Khajeh, H. Homayoun, E. Bozorgzadeh, A. Eltawil, and F. J. Kurdahi, "Reliability-aware placement in SRAM-based fpga for voltage scaling realization in the presence of process variations," in CODES+ISSS, pp. 257-266, 2011.

[23] A. Ghosh, R. M. Rao, C.-T. Chuang, and R. B. Brown, "On-chip process variation detection and compensation using delay and slew-rate monitoring circuits," in ISQED, pp. 815-820, 2008.

[24] C.-Y. Chen, H.-C. Shih, C.-W. Wu, C.-H. Lin, and P.-F. Chiu, "RRAM defect modeling and failure analysis based on march test and a novel squeeze-search scheme," IEEE Transactions on Computers, vol. 64, Jan. 2015

[25] S. Kirkpatrick, C. D. Gelatt, Jr., and M. P. Vecchi, "Optimization by simulated annealing," Science, vol. 220, pp. 671-680, 1983.

[26] H. Kuhn, "The hungarian method for the assignment problem," in NRLQ, pp. 8397, Mar. 1955. 